\title{
Fontan operation for hypoplastic left heart syndrome with absent aortic valve
}

\author{
Narutoshi Hibino, MD, ${ }^{a}$ Yorikazu Harada, MD, ${ }^{a}$ Takeshi Hiramatsu, MD, \\ Satoshi Yasukochi, MD, and Gengi Satomi, MD, ${ }^{\mathrm{b}}$ Nagano, Japan
}

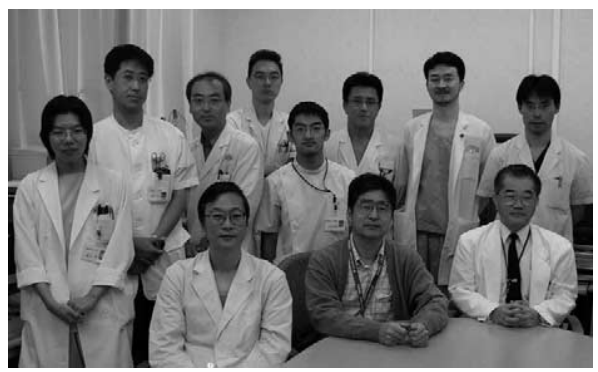

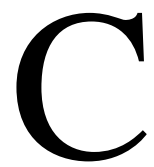

ongenital absence of aortic valve leaflets is an extremely uncommon complication of hypoplastic syndrome. We describe the first successful experience with a Fontan operation after the Norwood and bidirectional Glenn procedures for hypoplastic left heart syndrome with congenital aortic valve absence.

\section{Clinical Summary}

A 6-year-old boy was referred to our hospital after initially having cyanosis and respiratory distress at birth after an uneventful term pregnancy. On first admission, echocardiography showed mitral valve atresia and an absent aortic valve with a small and thick left ventricular cavity wall. Cardiac catheterization performed at 8 days of age had revealed a hypoplastic left ventricle with severe hypokinesis and severe aortic regurgitation. At 9 days of age, the patient underwent the Norwood procedure with a 4-mm polytetrafluoroethylene graft for a Blalock-Taussig shunt.

In the findings of the Norwood operation, the aortic annulus formed a mild narrowing with completely absent aortic valve. The aortic annulus was not closed because the coronary circulation would be maintained as a result of the severely hypokinetic small left ventricle. At the age of 6 months, the patient successfully underwent a bidirectional Glenn operation.

Cardiac catheterization after the Glenn procedure showed a gradually dilated left ventricle with improved contraction (Figure 1). Because the dilated ventricle had the possibility of coronary steal, a balloon occlusion test in the left ventricular outflow tract (LVOT) was performed during cardiac catheterization before the Fontan operation to determine the necessity of intervention to the aortic annulus and left ventricle. The echocardiographic results obtained during the balloon occlusion test showed that cardiac output measured on the basis of the flow velocity at the abdominal aorta had no relationship to the rate of occlusion in the LVOT. In

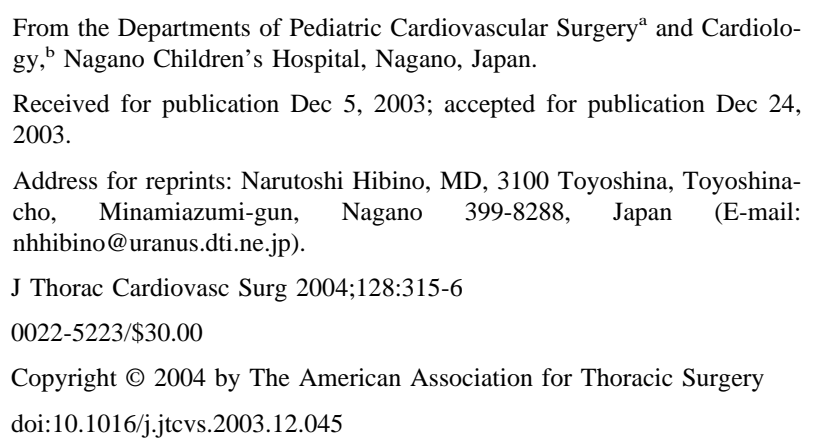

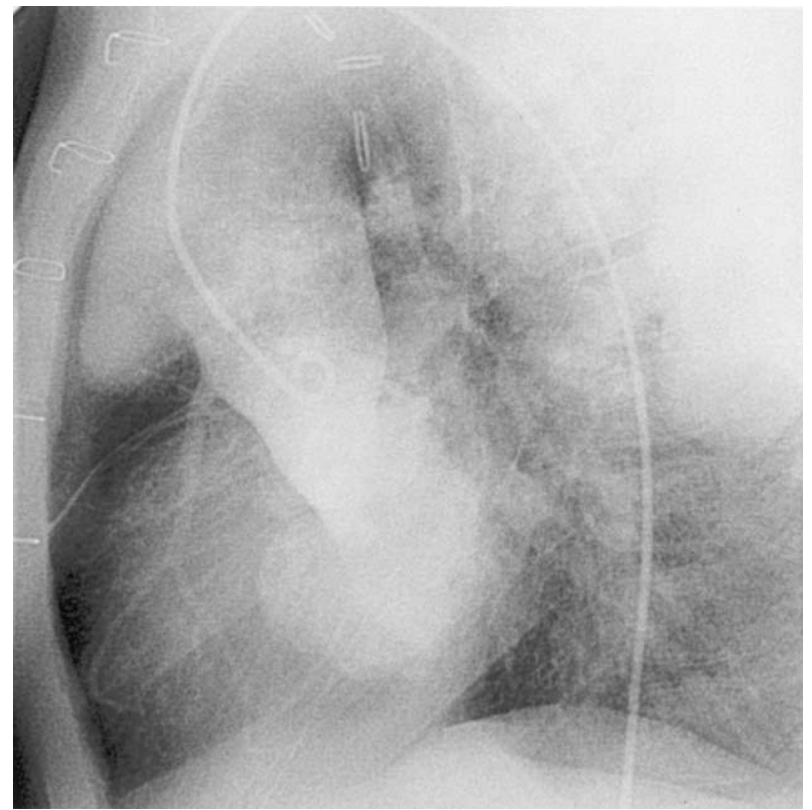

Figure 1. Left ventriculography after the Glenn procedure showed severe aortic regurgitation and a small left ventricle.

addition, the left ventricular end-diastolic pressure (LVEDP) was almost the same as the diastolic pressure in the ascending aorta, regardless of the occlusion in the LVOT. On the basis of these findings, we performed a total cavopulmonary connection procedure with an 18-mm polytetrafluoroethylene graft without any repair for the aortic annulus and left ventricle at the age of 6 years. The patient's postoperative course was uneventful. He has been followed up and has remained in New York Heart Association class I status, showing a strong ability for normal exercise.

\section{Discussion}

Absence of the aortic valve is an extremely uncommon congenital cardiac malformation. The rare incidence of this condition among newborns might be a consequence of poorly tolerated hemodynamics, resulting in spontaneous abortion. Toews and colleagues ${ }^{1}$ reported the first case of this anomaly associated with double outlet of the right ventricle. As far as we know, there have been 10 reported cases of absent aortic valve with a hypoplastic left ventricle. $^{2,3}$ However, all but our patient died after birth or during the neonatal period, regardless of palliative surgery. 
The cause of the absent aortic valve remains unknown. Proposed causative factors include a deficiency of primitive valvular tissue, abnormal ventriculoarterial septation, and early destruction of the valvular leaflets. Because all reported patients are male, $\mathrm{X}$-linked recessive inheritance or other X-linked factors have also been suggested.

The pathophysiology of this condition is complicated. Because the leaflets of the aortic valve are absent, free aortic valve insufficiency attributes to a persistent severely increased LVEDP, which promotes decreased coronary arterial perfusion pressure. Increased myocardial oxygen consumption caused by left ventricular hypertrophy, in combination with compromised coronary perfusion, produces a risk for ischemic heart disease.

As for echocardiographic findings, Bierman and coworkers ${ }^{4}$ noted pandiastolic regurgitation in the aorta during the fetal stage, as demonstrated with Doppler echocardiography, and observed only an immobile unrestrictive ridge on the proximal endocardial surface of this valveless aorta immediately after birth. Because of the rapid hemodynamic deterioration after delivery, a prenatal diagnosis would facilitate neonatal critical care.

In our case the baby survived during the first palliative stage ${ }^{5}$ because of the markedly diminished compliance of the left ventricle, which made it possible to maintain coronary perfusion. As the patient has grown, the left ventricle has developed with improved function. However, coronary perfusion might be sufficient because of the high LVEDP caused by mitral atresia and the absent aortic valve. In fact, cardiac catheterization showed aortic diastolic pressure and LVEDP to be almost the same. In addition, there was no pulmonary venous hypertension caused by mitral valve atresia or impaired oxygenation despite the increased left ventricular pressure.

We are observing the patient, paying special attention to the function of the left ventricle and the possibility of coronary steal syndrome.

\section{References}

1. Toews WH, Lortscher RH, Kelminson LL. Double outlet right ventricle with absent aortic valve. Chest. 1975;68:381-2.

2. Rouillard KP, Moore P, Silverman NH. Congenital absence of aortic valvar leaflets: a rare variant of the hypoplastic left heart syndrome. Cardiol Young. 2001;11:453-7.

3. Lin AE, Chin AJ. Absent aortic valve: a complex anomaly. Pediatr Cardiol. 1990;11:195-8.

4. Bierman FZ, Yeh MN, Swersky S, Martin E, Wigger JH, Fox H. Absence of the aortic valve: antenatal and postnatal two-dimensional and Doppler echocardiographic features. J Am Coll Cardiol. 1984;3: 833-7.

5. Harada Y, Takeuchi T, Satomi G, Yasukouchi S. Absent aortic valve: successful palliation in the neonate. Ann Thorac Surg. 1998;66:935-6.

\title{
Congenital tracheoesophageal fistulas presenting in adults: Presentation of two cases and a synopsis of the literature
}

\author{
J. Zacharias, FRCS (CTh), ${ }^{a}$ O. Genc, MD, ${ }^{\text {b }}$ and P. Goldstraw, FRCS, ${ }^{b}$ Newcastle upon Tyne and London, United Kingdom
}

$\mathrm{M}$ ore than $98 \%$ of tracheoesophageal fistulas (TEFs) are associated with atresia of the esophagus. ${ }^{1}$ These appear in the neonatal period, and early surgical treatment provides a satisfactory result. In the group in which a fistula is not associated with atresia, termed H-type fistula, symptoms might be minimal, and presentation might be delayed until adulthood. We report a single surgeon's experience of 2 patients with this unusual condition. Bronchoesophageal fistulas are more commonly reported, with more than

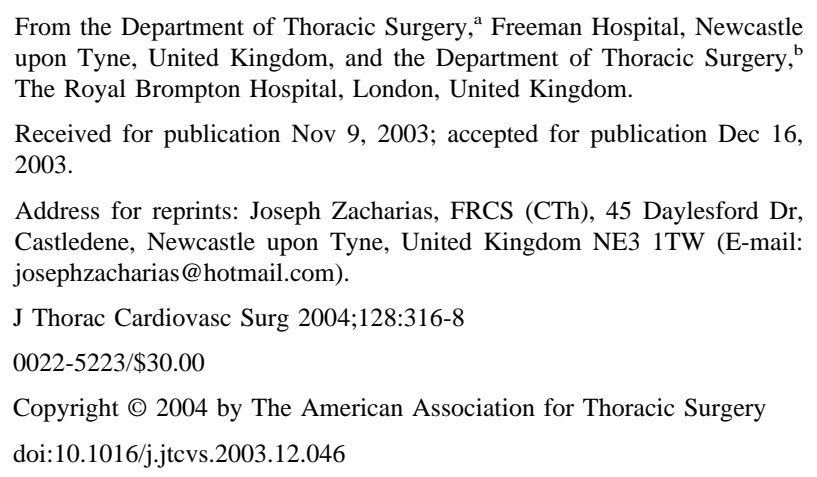

140 such cases detailed in the literature. ${ }^{2}$ We have reviewed and summarized the 14 reports in the English literature of congenital TEFs presenting in adults.

\section{Clinical Summary}

PATIENT 1. A 45-year-old female nonsmoker had a recurrent productive cough. It was worse over the past 2 years and was particularly troublesome at night. Examination with a fiberoptic bronchoscope showed a punctum in the posterior wall of the trachea 3.5 $\mathrm{cm}$ from the carina (Figure 1). No fistula was identified on barium swallow or esophagoscopy. A cine contrast study done while the patient was in the prone position confirmed the fistula between the esophagus and the lower trachea. At right-sided thoracotomy, the fistula was identified, divided, and repaired. A flap of parietal pleura was used to augment the esophageal repair. The postoperative course was uneventful, and at 1 year's follow-up, she remained symptom free.

PATIENT 2. A 55-year-old man had a history of persistent cough since childhood. Throughout his adult life, he had recurrent bouts of bronchitis. He also noticed that he often coughed or choked after swallowing liquids. He had right-sided pneumonia just 3 weeks before admission. A chest $\mathrm{x}$-ray film showed middle-lobe consolidation. Examination with a flexible bronchoscope showed a fistula 